\title{
Extreme-ultraviolet Stellar Characterization for Atmospheric Physics and Evolution mission: motivation and overview
}

\author{
Kevin France $\odot,{ }^{\text {a,* }}$ Brian Fleming $\odot,{ }^{\text {a }}$ Allison Youngblood, ${ }^{\text {a James Mason } \odot, ~}$ \\ Jeremy J. Drake, ${ }^{b}$ Ute V. Amerstorfer, ${ }^{\text {c }}$ Martin Barstow $\odot,{ }^{d}$

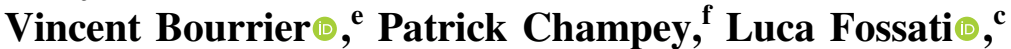 \\ Cynthia S. Froning ${ }^{\mathrm{g}}$ James C. Green, ${ }^{\text {h }}$ Fabien Grisé ${ }^{\mathrm{i}}$, \\ Guillaume Gronoff $\odot$, , Timothy Hellickson, ${ }^{a}$ Meng Jin,, ,I \\ Tommi T. Koskinen, ${ }^{\text {m }}$ Adam F. Kowalski, ${ }^{\text {,o }}$ Nicholas Kruczek, ${ }^{\text {a }}$ \\ Jeffrey L. Linsky, ${ }^{\text {p }}$ Sarah J. Lipscy, ${ }^{\mathrm{q}}$ Randall L. McEntaffer, ${ }^{\mathrm{i}}$ \\ David E. McKenzie, ${ }^{f}$ Drew M. Miles, ${ }^{i}$ Tom Patton, ${ }^{\text {a }}$ Sabrina Savage $\odot,{ }^{f}$ \\ Oswald Siegmund, ${ }^{\text {r }}$ Constance Spittler, ${ }^{\text {a Bryce W. Unruh, }}{ }^{\text {a }}$ \\ and Máire Volz \\ ${ }^{a}$ University of Colorado Boulder, Laboratory for Atmospheric and Space Physics, \\ Boulder, Colorado, United States \\ ${ }^{\mathrm{b}}$ Smithsonian Astrophysical Observatory, Cambridge, Massachusetts, United States \\ ${ }^{\mathrm{c}}$ Austrian Academy of Sciences, Space Research Institute, Graz, Austria \\ ${ }^{\mathrm{d}}$ University of Leicester, School of Physics \& Astronomy, Leicester, United Kingdom \\ eObservatoire Astronomique de l'Université de Genève, Versoix, Switzerland \\ ${ }^{\mathrm{f}} \mathrm{NASA} / \mathrm{Marshall}$ Space Flight Center, Huntsville, Alabama, United States \\ ${ }^{\mathrm{g}}$ University of Texas at Austin, McDonald Observatory, Austin, Texas, United States \\ ${ }^{\mathrm{h}}$ University of Colorado Boulder, Center for Astrophysics and Space Astronomy, Boulder, \\ Colorado, United States \\ iPennsylvania State University, Department of Astronomy \& Astrophysics, University Park, \\ Pennsylvania, United States \\ ${ }^{j}$ Science Systems and Application Inc., NASA Langley Research Center, Hampton, \\ Virginia, United States \\ ${ }^{\mathrm{k}}$ Lockheed Martin Solar \& Astrophysics Laboratory, Palo Alto, California, United States \\ ${ }^{1}$ SETI Institute, Mountain View, California, United States \\ ${ }^{\mathrm{m}}$ University of Arizona, Lunar and Planetary Laboratory, Tucson, Arizona, United States \\ ${ }^{\mathrm{n}}$ University of Colorado Boulder, National Solar Observatory, Boulder, Colorado, \\ United States \\ ${ }^{\circ}$ University of Colorado, Department of Astrophysical and Planetary Sciences, Boulder, \\ Colorado, United States \\ ${ }^{\mathrm{p}}$ University of Colorado and NIST, UCB 440, Joint Institute for Laboratory Astrophysics, \\ Boulder, Colorado, United States \\ ${ }^{\mathrm{q}}$ Ball Aerospace, Boulder, Colorado, United States \\ ${ }^{\mathrm{r}}$ University of California, Space Sciences Laboratory, Berkeley, California,
}

United States

\begin{abstract}
The Extreme-ultraviolet Stellar Characterization for Atmospheric Physics and Evolution (ESCAPE) mission is an astrophysics Small Explorer employing ultraviolet spectroscopy (EUV: 80 to $825 \AA$ and FUV: 1280 to $1650 \AA$ ) to explore the high-energy radiation environment in the habitable zones around nearby stars. ESCAPE provides the first comprehensive study of the stellar EUV and coronal mass ejection environments that directly impact the habitability of rocky exoplanets. In a 20-month science mission, ESCAPE will provide the essential stellar characterization to identify exoplanetary systems most conducive to habitability and provide a roadmap for NASA's future life-finder missions. ESCAPE accomplishes this
\end{abstract}

\footnotetext{
*Address all correspondence to Kevin France, kevin.france@colorado.edu
} 
France et al.: Extreme-ultraviolet Stellar characterization for atmospheric physics and evolution mission...

goal with roughly two-order-of-magnitude gains in EUV efficiency over previous missions. ESCAPE employs a grazing incidence telescope that feeds an EUV and FUV spectrograph. The ESCAPE science instrument builds on previous ultraviolet and $\mathrm{x}$-ray instrumentation, grazing incidence optical systems, and photon-counting ultraviolet detectors used on NASA astrophysics, heliophysics, and planetary science missions. The ESCAPE spacecraft bus is the versatile and high-heritage Ball Aerospace BCP-Small spacecraft. Data archives will be housed at the Mikulski Archive for Space Telescopes. () The Authors. Published by SPIE under a Creative Commons Attribution 4.0 International License. Distribution or reproduction of this work in whole or in part requires full attribution of the original publication, including its DOI. [DOI: 10.1117/1 .JATIS.8.1.014006]

Keywords: small explorer; extreme ultraviolet; exoplanets; flares and coronal mass ejections; spectroscopy.

Paper 21082 received Aug. 6, 2021; accepted for publication Jan. 14, 2022; published online Feb. 12, 2022.

\section{Introduction}

Owing to their large number and strong atmospheric impacts, extreme ultraviolet (EUV) photons are the primary agent driving atmospheric escape on planets orbiting cool stars $(\mathrm{F}, \mathrm{G}, \mathrm{K}$, and $\mathrm{M}$ stars; $\sim 2$ to 0.1 solar masses). The stability of Earth-like atmospheres critically depends on the EUV irradiance. ${ }^{1,2}$ Higher EUV flux from the young Sun $^{3}$ could have led to $10 \times$ greater oxygen loss rates and 90× greater carbon loss rates on the early Martian atmosphere, compared to present day levels, by increasing the suprathermal or "hot" population of these atoms. ${ }^{4}$ On highly irradiated planets, the outflow is sufficiently rapid that heavier atmospheric species (e.g., N, C, $\mathrm{O}, \mathrm{Mg}$, and $\mathrm{Fe}$ ) can be dragged along through collisions with the lighter hydrogen, as observed even on hot Jupiters. ${ }^{5-11}$ Free electrons produced by stellar EUV photons can also attain altitudes much greater than ions, producing an ambipolar electric field that leads to a non-thermal ionospheric outflow in $\mathrm{O}^{+}$and $\mathrm{N}^{+}$winds. ${ }^{12-15}$ Consequently, a major open question as astronomers embark on the spectral characterization of rocky exoplanets in the coming decade with James Webb Space Telescope (JWST) is whether terrestrial atmospheres can survive the radiation environments around other stars. ${ }^{16}$

Previous EUV observatories have lacked the sensitivity to survey exoplanet host stars in this spectral band, and as a result, widely discrepant techniques have been employed to approximate the critical stellar inputs into exoplanet atmosphere models. In this paper, we present the motivation for and the mission overview of the extreme-ultraviolet Stellar characterization for atmospheric physics and evolution (ESCAPE) mission. ESCAPE explores the habitable zone (HZ) radiation environment around more than 200 nearby stars, filling the observational gap in planetary atmosphere evolution models, models that enable us to predict which star-planet systems are most promising for developing and maintaining habitable conditions. These nearby stellar systems make up the target list for future NASA missions focused on spectroscopic characterization of rocky planets.

ESCAPE investigates stars of spectral types F, G, K, and M to be responsive to all terrestrial exoplanet characterization missions currently in implementation or formulation. Terrestrial exoplanet research with JWST in the 2020s and the Origins Space Telescope (OST) or an infrared (IR) probe in the $2030 \mathrm{~s}$ are best suited to study transiting exoplanets around M dwarfs. Direct detection missions of the 2030 and 2040s, such as Habex, LUVOIR, and the 6-m UVOIR mission recently recommended by the Astro2020 Decadal Survey will be optimized for exo-Earths orbiting more massive F, G, and K stars. ESCAPE 's observing program provides the stellar EUV context for whatever technical and programmatic approach the community adopts to identify and characterize habitable worlds. The ESCAPE data set will allow the astronomical community to predict the most promising star-planet systems for life detection missions in the coming decades, establishing a roadmap for future observing resources to explore habitable worlds. 


\section{Science Objectives and Implementation}

ESCAPE's primary science goal is to identify and understand those star-planet systems that are conducive to the formation of habitable environments. The ESCAPE mission addresses this goal by answering three key science questions:

1. What is the EUV irradiance in the HZ?

2. How does stellar EUV irradiance evolve in time?

3. What are the properties of stellar coronal mass ejections (CMEs)?

ESCAPE addresses these science questions through a comprehensive observing program supported by state-of-the-art planetary atmosphere models that take ESCAPE 's observables as inputs and are used to estimate atmospheric mass loss rates from representative terrestrial planets. The ESCAPE instrument: (1) achieves $\approx 25 \times$ to $100 \times$ the EUV efficiency of previous missions, resolving order-of-magnitude uncertainties on exoplanet radiation environments; (2) explores EUV variability from flares, stellar rotation, and stellar evolution with its photoncounting detector, monitoring survey, and sample of F, G, K, and M stars with a range of ages; and (3) executes the first survey of stellar CMEs using techniques validated in "Sun-as-a-star" measurements by NASAs Solar Dynamics Observatory-EUV Variability Experiment EVE (SDO-EVE) instrument, providing direct constraints on exoplanet particle environments.

\section{High-Energy Radiation Environments: EUV Photons, Variability, and CMEs}

\subsection{Stellar EUV Irradiance}

Optical and near-infrared photons heat the surface and troposphere. NUV (1800 to $3200 \AA$ ), FUV (912 to $1800 \AA$ ), and x-ray (5 to $100 \AA$ ) photons are absorbed in the middle and upper atmosphere where they photo-dissociate molecules and ionize heavy elements. EUV photons (100 to $911 \AA$ ) are absorbed high in the atmosphere (i.e., in the thermosphere) where they ionize atoms and molecules. Liberated electrons collisionally heat the surrounding gas, increasing the scale height of the atmosphere, driving ambipolar ion mass loss, and potentially leading to the formation of a hydrodynamic outflow. Rapid atmospheric loss can lead to both desiccation and the build-up of abiotic $\mathrm{O}_{2}$ atmospheres that complicate biosignature searches. $^{17-19}$

EUV photons are the key drivers for atmospheric mass-loss for three primary reasons: (1) EUV photons are absorbed in the highest (lowest density) layers of the atmosphere, where radiative losses (proportional to density squared) are minor and the heating efficiency is highest, (2) the ionization cross-sections for dominant upper atmospheric atomic species (e.g., H, $\mathrm{N}$, and $\mathrm{O}$ ) peak at EUV wavelengths, and (3) there are many more EUV photons than $\mathrm{x}$-rays available on all types of stars to drive this heating. In the quiet Sun, the EUV/x-ray photon production ratio is $\sim 90 .{ }^{20}$ For optically inactive early $\mathrm{M}$ dwarfs, the EUV/X-ray photon ratio is $\sim 40,{ }^{21}$ and even for an active late $\mathrm{M}$ dwarf like Proxima Cen, the EUV/X-ray photon ratio is $\sim 16$ (Fig. 1).

The discovery of rocky planets in the HZs of nearby stars, e.g., Proxima Cen b and the TRAPPIST-1 planets, has motivated new atmospheric mass loss calculations that highlight the need for improved EUV irradiance data to estimate their long-term habitability. Garcia-Sage et al. ${ }^{28}$ and Airapetian et al. ${ }^{29}$ presented studies of EUV-driven ion loss from Earth-like planets orbiting M dwarfs. They found mass loss rates several orders of magnitude higher than that of present-day Earth for EUV fluxes 10 to 20 times the current day EUV solar irradiance. Their models indicate that elevated EUV fluxes, augmented by persistent flares or the long pre-mainsequence phase of $\mathrm{M}$ dwarfs, could effectively render some Earth-like planets around $\mathrm{M}$ dwarfs devoid of an atmosphere, in the absence of internal or external resupply of volatiles. A factor of ten in the uncertainty of incident EUV flux can lead to three orders of magnitude in the uncertainties of ion escape rates, ${ }^{28}$ fundamentally shifting our predictions for which exoplanets are the best candidates for long-lived habitable atmospheres. 
(a)

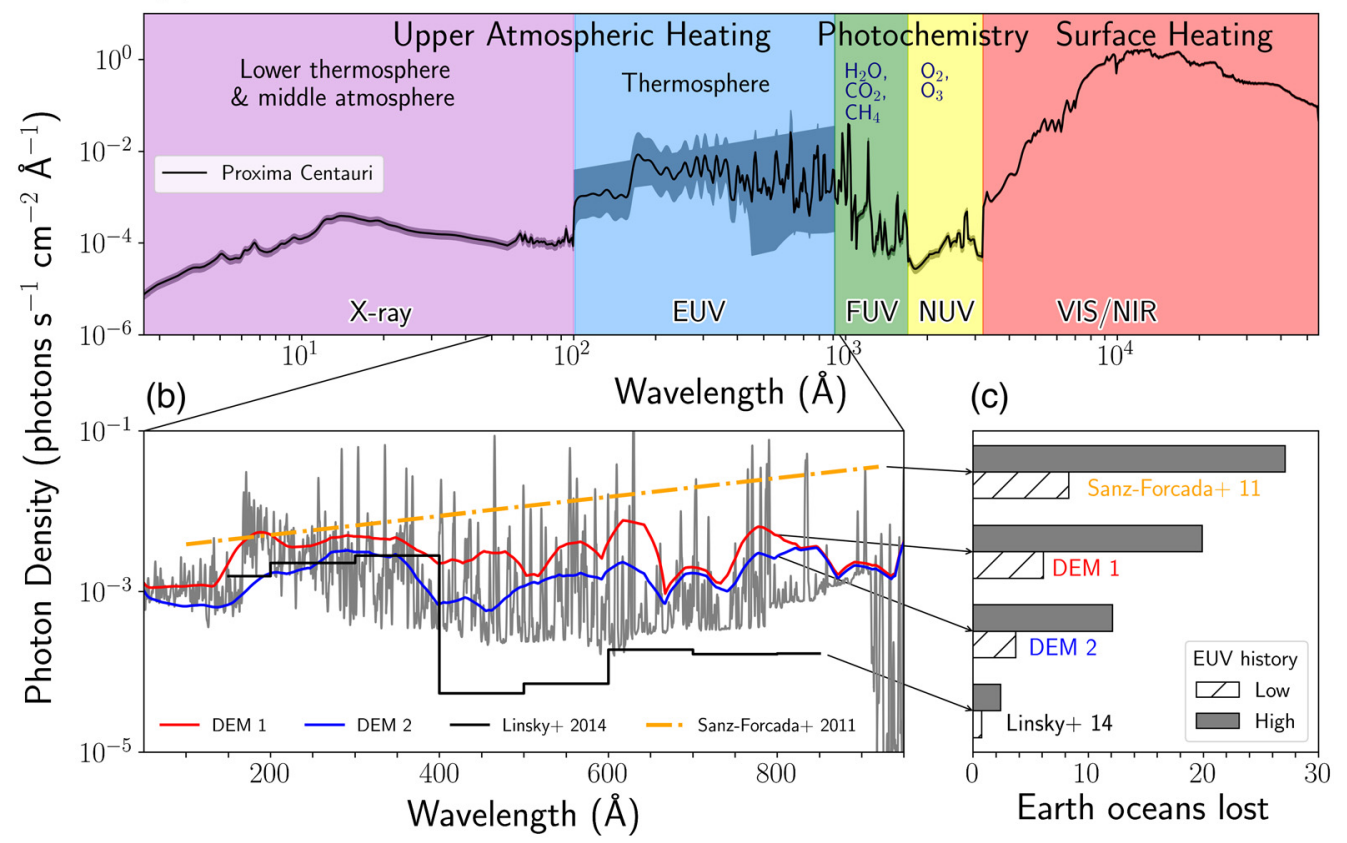

Fig. 1 (a) Composite spectrum of the nearby planet-hosting $M$ dwarf Proxima Cen, adapted from France et al. $(2016)^{22}$ and Loyd et al. (2016) ${ }^{23}$ The width of the shaded region indicates the approximate uncertainty on the intrinsic flux level. (b) Different reconstructions of the EUV spectrum of Proxima Cen show factors of 3 to 100 flux discrepancies: differential emission measure models in red, blue, and gray (Drake et al. 2020); ${ }^{24}$ scaling relations based on only FUV data in black (Linsky et al. 2014) ${ }^{25}$ and only x-ray data in orange (Sanz-Forcada et al. 2011). ${ }^{26}$ (c) The corresponding atmospheric hydrogen mass lost from an Earth-like planet (in units of Earth oceans; 1 Earth ocean $=1.4 \times 10^{24} \mathrm{~g}$ ) from $10 \mathrm{Myr}$ to 4.8 Gyr for high (shaded gray) and low (hatched) EUV histories of typical M dwarfs (Ribas et al. 2017). ${ }^{27}$

Figure 1 shows the results of analogous thermal atmospheric escape calculations for Proxima Cen $\mathrm{b}$ under different plausible EUV radiation strengths and histories. Model calculations yield a factor of $\sim 30$ spread in the total atmospheric mass loss from the planet over time, driven almost entirely by what assumptions are used to estimate the current EUV flux and its evolution earlier in the star's history.

Despite their importance, the EUV luminosity, flare rate, and evolution of other stars are almost completely unconstrained and can only be empirically determined with direct measurements of the EUV flux and temporal variability of a sample of stars with a range of ages and activity levels. Existing EUV spectra of exoplanet host stars are scarce. The only previous EUV astronomy mission, the extreme ultraviolet explorer (EUVE; Bowyer ${ }^{30}$ and Malina 1991), obtained spectra of $\sim 15 \mathrm{cool}$ main sequence stars, including $5 \mathrm{M}$ dwarfs. EUVE's observations were heavily biased toward the most active stars. The very modest $\leq 2 \mathrm{~cm}^{2}$ peak effective area of the EUVE spectrometers precluded useful spectroscopic observations of stars with more solarlike activity, except for the nearby $\alpha$ Cen system and the F4 subgiant Procyon. ${ }^{31,32}$ No observed EUV spectra exist of the optically inactive $\mathrm{M}$ dwarfs (defined here as $\mathrm{Ca}$ II $\mathrm{H}$ and $\mathrm{K}$ equivalent widths $<1 \AA$ ) $, 22,33$ which will be optimal for atmospheric studies with JWST, 30-m telescopes, and the Origins Space Telescope. The lack of direct EUV data hampers our ability to understand how habitable atmospheres evolve with time and to design truly definitive biosignature searches.

\subsection{EUV Evolution and Flares}

Solar EUV emission varies by factors of up to $\sim 100$ on minute timescales due to flares and by factors of $\sim 6$ on year timescales due to the solar cycle. ${ }^{34}$ Different emission lines and continua vary by different factors on all timescales, whereas connections between the flare energy and 
frequency distribution of white light flares [such as those observed with Kepler/K2 and Transiting Exoplanet Survey Satellite (TESS)] and UV flares remain poorly constrained by observation and theory. ${ }^{35-38}$ Estimates of the EUV response of the Great AD Leo Flare of $1985^{39}$ observed with the International Ultraviolet Explorer indicate that the atmospheres of planets orbiting very active stars with frequent flares never achieve a steady state because the timescales for atmospheric recovery are much longer than the time between successive flares. ${ }^{40}$ EUVE provided constraints on flare frequencies for a small sample of young, active stars, ${ }^{41}$ however, the lack of direct constraints on EUV flare activity on older stars (e.g., France et al. $2020)^{42}$ hinders our ability to model the stability of potentially habitable atmospheres over timescales required for the initial development of surface life ( 1.7 Gyr; Jones and Sleep) ${ }^{43}$ ESCAPE employs a photon-counting detector to record all observations in a "time-tagging" mode that provides temporal resolution $(<20 \mathrm{~s}$, limited by photon statistics, the instrumental timing resolution is $<0.1 \mathrm{~s}$ ) higher than the characteristic UV flare duration on low-mass stars ( $\sim 300$ s; Hawley et al. 2003; Loyd and France 2014; Loyd et al. 2018a). ${ }^{44-46}$

Characterization of the EUV flare frequency and spectral variability on stellar evolutionary timescales requires (1) directly collecting EUV spectra over long enough temporal baselines to monitor variability and (2) a broad sample of stars with a range of masses and ages (activity levels, e.g., West et al.). ${ }^{47}$ The required monitoring observations cannot be collected from the ground because of the lack of optical features whose behavior is closely linked to the EUV emission. For example, inactive $\mathrm{M}$ dwarfs that show little optical lightcurve variation at the $<1 \%$ level can display factors of $\sim 50$ increases in the FUV and $\mathrm{x}$-ray, ${ }^{22,46}$ and we expect the behavior to be similar at EUV wavelengths. ESCAPE employs a dedicated monitoring program to sample the flare frequency and energy distribution for individual systems across spectral classes and ages (Sec. 5).

\subsection{Detecting Stellar CMEs}

High-energy particles have a major influence on the evolution of exoplanetary atmospheres, both through the actions of stellar winds and impulsive events (e.g., CMEs). While stellar winds play a part in atmospheric escape, CMEs are thought to be the dominant source of long-term instability in planetary atmospheres. ${ }^{48-50}$ The influence of CMEs depends strongly on their frequency and ability to break out of coronal magnetic confinement; ESCAPE will measure the frequencies of CMEs on solar-type stars and search for CME breakout from M dwarfs.

While the main source of heat in the upper atmosphere of planets is the EUV flux, Joule heating and particle precipitation heating could dominate the chemical impact of impulsive events (e.g., Segura et al. $2010^{35}$ and Tilley et al. 2019) ${ }^{51}$ CMEs deliver heat to the upper atmospheres of orbiting planets through charge exchange reactions. ${ }^{52}$ Pickup and sputtering processes can also be major sources of atmospheric loss from direct particle precipitation and stellar wind enhancements due to CMEs. CMEs contribute to atmospheric mass loss on solar system planets $^{53,54}$ and to space weather, leading several authors to propose an expanded definition of the HZ that takes into account space weather impacts (e.g., Airapetian et al. 2017 and 2020) ) $^{15,29}$ as more meaningful than the traditional liquid water $\mathrm{HZ}$.

About $\sim 90 \%$ of large (X-class) solar flares are associated with CME-like particle eruptions, ${ }^{55}$ however, this connection has not been borne out with recent observations of stellar flares. ${ }^{56-58}$ While the EUV irradiance is increased by a large flare rate, CMEs and accelerated particles may have much greater impacts on atmospheric photochemistry and stripping than the flare radiation itself. 35,49

For the Sun, we are able to observe CMEs directly with coronagraphs and in situ measurements. These traditional methods are not feasible for stellar CMEs in the near-term (although see Haisch et al. and Moschou et al.). ${ }^{59,60}$ However, other techniques for detecting solar CMEs have been developed. Harra et al ${ }^{61}$ reviewed these techniques and determined that coronal dimming, described below, is the only feature consistently associated with CMEs that can be employed to detect and quantify these events on other stars.

Solar coronal dimming studies have primarily relied upon spatially resolved EUV images to characterize the transient voids left behind in the corona as a CME departs, giving rise to the observed flux dimming (e.g., Sterling and Hudson, Aschwanden et al., Reinard and Biesecker; 
(a)

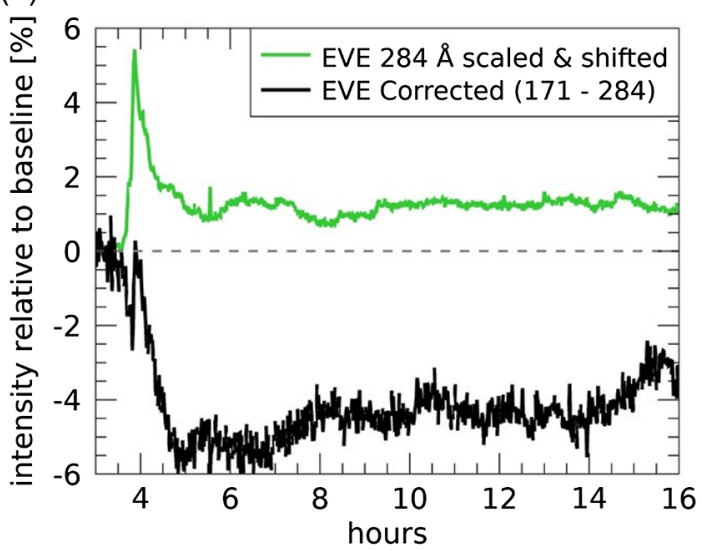

(b)

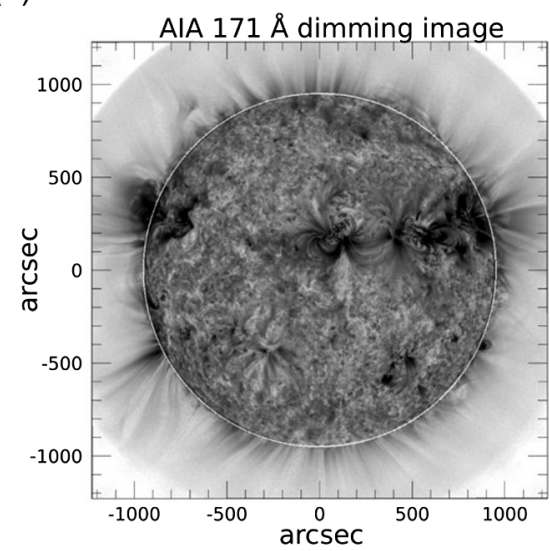

Fig. 2 (a) Observation of coronal dimming in full-disk solar spectra and (b) angularly resolved filter imaging. The spectrally resolved lightcurves from SDO-EVE show flaring lines ( $F e$ XV $284 \AA$, green) and the corrected Fe IX $171 \AA$ dimming profile in black (Mason et al. 2016). ${ }^{68}$ ESCAPE is sensitive to similar events on $F, G$, and $K$ dwarfs to a distance of $6 \mathrm{pc}$. More than 90 candidate events are expected in the DEEP survey (Sec. 5.2).

Dissauer et al. 2018a,b) ${ }^{62-66}$ The SDO-EVE, Woods et al. 2012, ${ }^{34}$ demonstrated that dimming can also be characterized in disk-integrated EUV spectra. ${ }^{67-70}$ Similar dimming events have recently been reported in x-ray lightcurves of active stars and an archival EUVE spectrum of the young K star AB Dor. ${ }^{71}$ ESCAPE employs the same Sun-as-a-star stellar CME characterization techniques validated on SDO-EVE (Fig. 2) to measure the frequency of CMEs on nearby stars for the first time.

The dimming light curve also contains information about the kinematics of the CME that produced it. Solar dimming lightcurves have been calibrated by in-situ proton measurements and coronagraphic imaging of CMEs. The depth of the dimming event is directly related to the mass of the ejected CME (the dispersal of the quiescent corona greatly reduces the emission measure, which scales as density squared), whereas the slope of the dimming lightcurve indicates the CME propagation velocity ${ }^{68,69}$ Therefore, observations of coronal dimming events on solar-type stars can provide direct constraints on the physical properties of the CME.

\section{Interstellar Attenuation and Flux Reconstruction}

Dust and molecular hydrogen extinction is negligible within the local cavity (Lehner et al. $;^{72}$ also historically referred to as the local bubble), meaning that the only significant sources of line-ofsight opacity (or, reddening) are neutral and low-ionization atomic gases. ${ }^{73}$ The ESCAPE FUV bandpass covers 1280 to $1650 \AA$ (excluding the strong Ly $\alpha$ line); the only significant sources of interstellar opacity in the FUV band are neutral oxygen and singly ionized carbon and silicon, which impact measurements of low-ionization lines in ESCAPE FUV range (e.g., C II). In ESCAPE EUV range, neutral hydrogen ( $\mathrm{H} \mathrm{I}$ ), neutral helium (He I), and ionized helium (He II) contribute, with the $\mathrm{H}$ I column density being the controlling parameter for the total line-of-sight EUV extinction (Fig. 3). Despite the physical complexity of the local ISM, integrated H I column density rarely exceeds $10^{18.4} \mathrm{~cm}^{-2}$ within $30 \mathrm{pc}$ of the $\mathrm{Sun}^{74}$ and do not reach $10^{19} \mathrm{~cm}^{-2}$ routinely until $d>80 \mathrm{pc}$, beyond ESCAPE's baseline mission target distance.

ESCAPE employs three techniques to measure or estimate the interstellar hydrogen column density $[\mathrm{N}(\mathrm{HI})]$ toward its targets and therefore to correct for the ISM attenuation in its spectra. First, high-fidelity direct measurements of N(HI) are routinely acquired with the HST-STIS instrument (or archival data from HST-GHRS). Direct Lyman- $\alpha$ measurements of $\mathrm{N}(\mathrm{HI})$ yield uncertainties of $<0.1$ dex (see, e.g., Table 2 of Wood et al. 2005; $;^{74}$ Tables 2 and 3 of Youngblood et al. 2016).$^{75}$ Second, an all-sky $\mathrm{n}(\mathrm{HI})$ map created from ensemble archival N(HI) measurements has been created and subsequently validated by comparing new observations from Hubble Space Telescope (HST) ${ }^{76}$ against the predicted N(HI) from the all-sky map, finding agreement between 


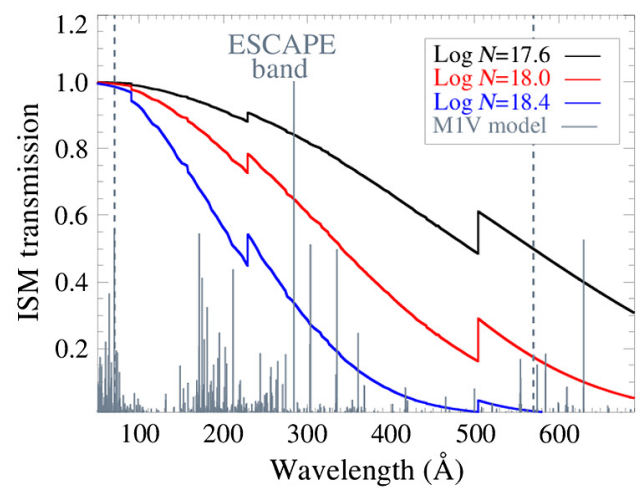

Fig. 3 Transmission of the local interstellar medium for $\mathrm{H}$ I column densities typical of stars inside 30 pc (10 $17.6-18.4 \mathrm{~cm}^{-2}$; Wood et al. 2005). ${ }^{74}$ These curves demonstrate that the ISM is more than $20 \%$ transparent to EUV photons over most of the ESCAPE G20 and G40 bandpasses for most stars inside $30 \mathrm{pc}$.

direct observation and all-sky model prediction of $\leq 0.25$ dex. This level of interstellar column uncertainty yields absolute flux uncertainties $\leq 35 \%$ for wavelengths shorter than $300 \AA$ and column densities typical of the local ISM within $30 \mathrm{pc}\left(\leq 10^{18.4} \mathrm{~cm}^{-2}\right)$. Finally, these empirical techniques are augmented by three-dimensional ISM models based on empirical studies of the local ISM and publicly available to the community (Ref. ${ }^{73}$ ). The ISM models of Redfield and Linsky (Ref. ${ }^{73}$ ) predict the line-of-sight column density through the local ISM for an arbitrary look direction.

Stars within $30 \mathrm{pc}$ encompass almost all $\mathrm{HZ}$ planets that are candidates for spectroscopic characterization and biomarker detection (LUVOIR Team Final Report ${ }^{77}$ ). ESCAPE has the sensitivity to meet the required signal-to-noise and temporal resolution to address the key science questions (Sec. 2) for F, G, K, and active M stars out to $30 \mathrm{pc}$ and inactive stars earlier than M5 out to $8 \mathrm{pc}$. Thus, the interstellar medium is not the limiting factor in our ability to study EUV emission from the most important exoplanet hosts.

ESCAPE obtains accurate measurements of the 80 to $560 \AA$ spectrum of nearby cool stars in the primary EUV channels. The 570 to $911 \AA$ flux is also important for exoplanet heating calculations as this band overlaps with the peak of the ionization cross-sections of atomic and molecular hydrogen. However, attenuation by the local ISM in the 570 to $911 \AA$ region prevents observations from all but the very nearest stars with the lowest ISM column density sightlines.

ESCAPE takes a two-tiered approach to measuring these important intermediate EUV wavelengths. (1) ESCAPE's simultaneous coverage of coronal and transition region lines from 80 to $560 \AA$ and transition region and chromospheric lines in the FUV channel from 1300 to $1650 \AA$ (e.g., C II, Si IV, and C IV) provide robust constraints on differential emission measure calculations (DEM; see, e.g., Drake et al. $2020 ;{ }^{24}$ Duvvuri et al. ${ }^{78}$ and references therein), allowing us to calculate accurate stellar fluxes in the 570 to $911 \AA$ region. (2) ESCAPE includes a lowresolution mode covering 600 to $825 \AA$ with sufficient sensitivity to directly measure stellar emission in this bandpass, including the important $\mathrm{H}$ I Lyman continuum, for $\sim 10$ stars in the DEEP survey (The 565 to $600 \AA$ and 825 to $911 \AA$ regions are avoided due to bright He I $584 \AA$ and O II $834 \AA$ geocoronal airglow emission, respectively.).

\section{ESCAPE Science Surveys: SEEN and DEEP}

\subsection{Stellar EUV ENvironments Survey}

The Stellar EUV ENvironments (SEEN) survey measures the 80 to $1650 \AA$ A irradiance and time variability for 200 stars with absolute photometric uncertainty $<40 \%$ in the 20 month baseline mission. The photometric accuracy requirement is selected to remove the stellar flux as a dominant source of uncertainty in atmospheric escape calculations. ${ }^{16}$ We require the sensitivity and spectral resolution to separate stellar emission lines and measure variability in individual spectral 


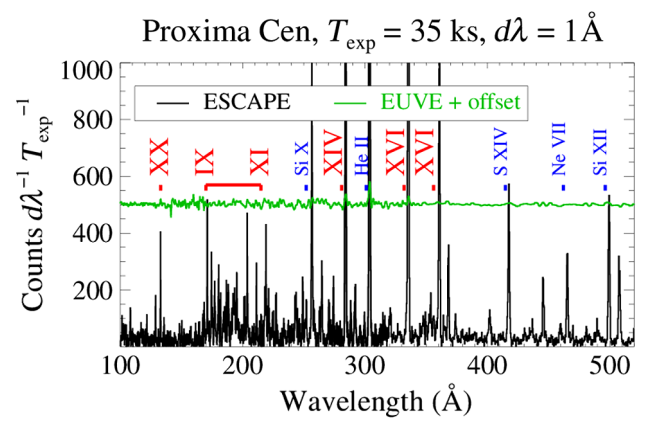

Fig. 4 A simulated ESCAPE SEEN spectrum of Proxima Cen is shown (black). The 77ks EUVE exposure of Proxima Cen (green, offset by 500 counts) resulted in tentative detections of $\sim 5$ stellar emission lines. ESCAPE achieves a line-integrated $\mathrm{S} / \mathrm{N}$ of $\sim 41$ at $\mathrm{Fe}$ IX $171 \AA$ A in $35 \mathrm{ksec}$. Prominent iron ionization states are labeled in red and other species in blue.

features. These requirements drive the telescope size and grazing incidence design to maintain high throughput at EUV wavelengths (Sec. 6). To illustrate the comparison of a SEEN survey observation with archival data available from EUVE, we show a simulated raw spectrum of Proxima Cen in Fig. 4.

About $\sim 20$ flares are required to develop a flare-frequency distribution with $\sim 50 \%$ logarithmic rate accuracy (e.g., the rate constant of the flare-frequency power-law distribution). To determine the required SEEN survey duration that effectively constrains the flare rates of $F, G, K$, and M dwarfs, we estimate the EUV flare rate based on the FUV flare-frequency distribution of Loyd et al. (2018a) ${ }^{79}$ and the FUV-to-EUV scaling relations from France et al. (2018). ${ }^{80}$

The flare frequency distribution is a power law, so more low energy flares are expected than high energy flares. Taking an intermediate activity $\mathrm{M}$ dwarf at $10 \mathrm{pc}$ as our fiducial system, we find flares with total Fe IX $171 \AA$ energy $E(\mathrm{Fe}$ IX $) \geq 5 \times 10^{29} \mathrm{erg}$ produce a 5- $\sigma$ flare detection with ESCAPE (assuming a typical 5-min M dwarf UV flare duration). This fiducial flare corresponds to a factor of $\sim 15$ brightening, typical for transition region flares observed on low-mass stars. ${ }^{4,46,79}$

From the estimated EUV flare rate, we find that in a $35 \mathrm{ksec}$ SEEN observation, we expect to detect 9 flares with $15 \times$ brightening $E(\mathrm{Fe} I X)=10^{29.75} \mathrm{ergs} ; 3$ flares with $50 \times$ brightening $E(\mathrm{Fe} \mathrm{IX})=10^{30.25}$; and 1 flare with $100 \times$ brightening $E(\mathrm{Fe} \mathrm{IX})=10^{30.55}$. To assemble statistically significant EUV flare-frequency distributions ( $\geq 20$ flares), we will group 3 to 5 stars with similar mass and age properties. Flaring rates and fluxes of young $\mathrm{G}$ and $\mathrm{K}$ stars are comparable to intermediate activity $\mathrm{M}$ stars; $;{ }^{15,81}$ therefore, we expect young $\mathrm{G}$ and $\mathrm{K}$ stars to be well represented by the inactive $\mathrm{M}$ dwarf calculations presented above.

ESCAPE measures the critical pre-main sequence history of M dwarf EUV luminosities, ${ }^{82,83}$ as well as the evolution of the EUV luminosity of F, G, and K stars (e.g., Johnstone et al. 2021; Fig. 5). The SEEN Survey covers a range of ages of each spectral type, spanning pre-mainsequence stars in the TW Hya and $\beta$ Pic moving groups (ages $<30 \mathrm{Myr}$ ), intermediate age clusters such as the AB Dor and the Hyades (hundreds of Myr; Schneider et al.), ${ }^{84}$ and "field" age stars comparable to the Sun (Gyr, based on gyrochronolgy and UV/optical activity indicators). Each spectral type is broken down into approximate "early" (subclass 0 to 4 ) and "late" (subclass 5 to 9 ) subgroups, e.g., [early F type stars (F0V to F4V), late-F type stars (F5V to F9V), early G type stars (G0V to G4V), etc.]. The SEEN survey data allow us to assess how similar the EUV outputs of two stars of comparable mass and age are, and importantly, allows us to develop a range of EUV flux estimates for a given set of basic stellar parameters that provides empirical bounds for studies of exoplanets beyond the lifetime of the ESCAPE mission.

A full 200-star target list that meets these requirements at sufficient $\mathrm{S} / \mathrm{N}$ is shown in Figs. 5 and 6. About 41 of the baseline stars host known planets (with 85 total planets, 15 of which are in the $200 \mathrm{~K}$ to $330 \mathrm{~K}$ temperate zone). While this target list satisfies ESCAPE's science objectives, ongoing results from TESS and Characterising Exoplanets Satellite (CHEOPS) will inform the final target list that will be finalized prior to launch. Finally, it should be noted that exoplanet populations statistics tell us that many of ESCAPE's "planetless" target stars likely host planets

J. Astron. Telesc. Instrum. Syst. $\quad$ 014006-8 Jan-Mar 2022 • Vol. 8(1) 
France et al.: Extreme-ultraviolet Stellar characterization for atmospheric physics and evolution mission...

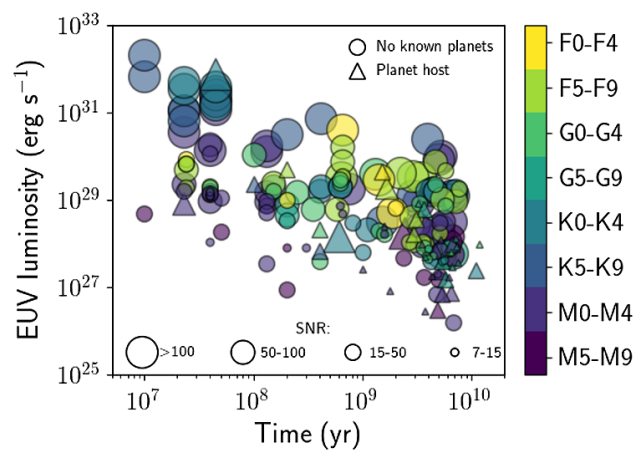

Fig. 5 Estimated range of the EUV radiation field on stellar evolutionary timescales, characterized in ESCAPE 's SEEN survey. The EUV luminosity is the estimated 90 to $360 \AA$ intrinsic luminosity and the symbol sizes represent the integrated S/N of Fe IX in a $35 \mathrm{ksec}$ SEEN observation. Stars with confirmed exoplanets are shown as triangles. This plot shows our current 200 star target list, with resolution in both age and mass (color-coded).

(a)

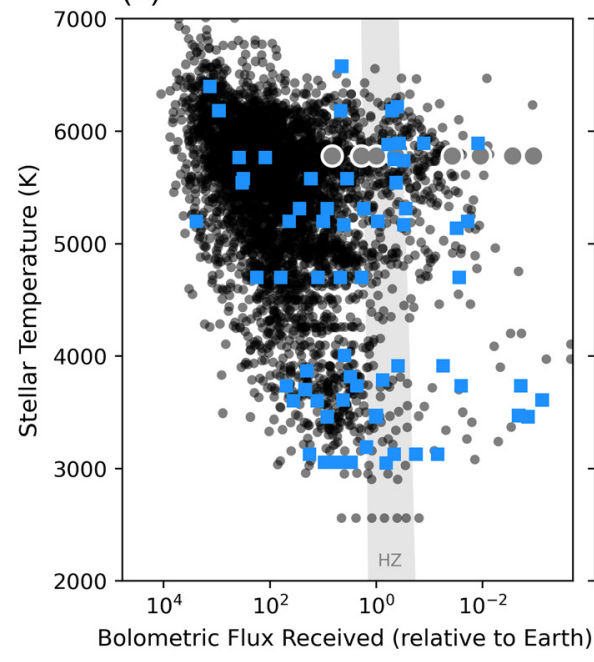

(b)

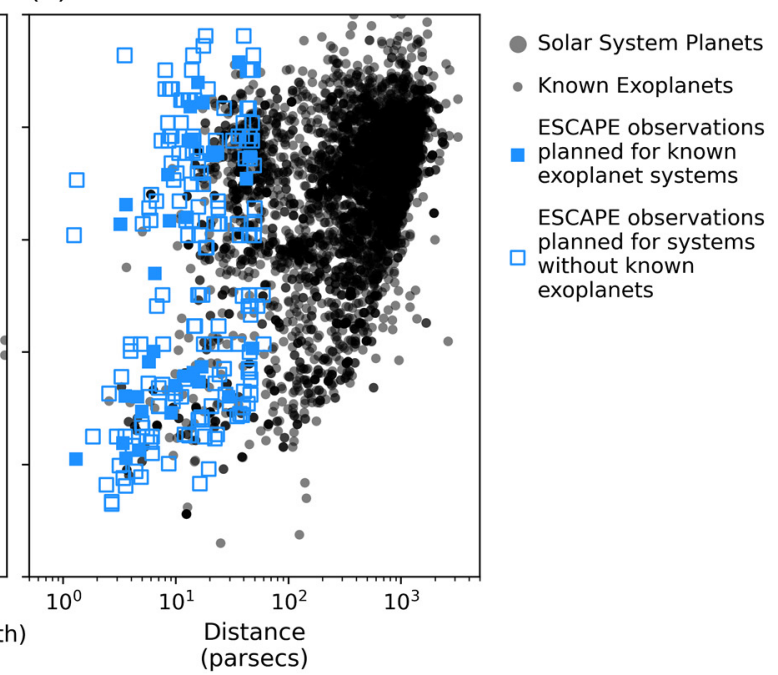

Fig. 6 The ESCAPE target list consists of 41 known exoplanet host stars, and is representative of the underlying known exoplanet population. (a) The known exoplanet population is shown as bolometric flux received against the host star's effective temperature. (b) The known exoplanet population is shown as distance against stellar effective temperature. Solar system planets are shown as large gray/white circles, exoplanets are shown as small grey circles, and ESCAPE planned targets are blue squares (filled = known exoplanets, empty = no exoplanets yet known). An approximate estimate for the temperate zone (recent-Venus to early Mars; Kopparapu et al. $2013)^{87}$ is shown as the gray shaded region.

that are yet to be detected (Dressing and Charbonneau; Stark et al. and references therein). ${ }^{85,86}$ Future direct-detection missions (e.g., LUVOIR and Habex) will both find and characterize these Earth-like planets around nearby solar-type (F, G, and K) stars. ESCAPE's SEEN survey ensures that when planets are directly imaged around these stars and spectroscopic characterization begins, the fundamental stellar constraints on the long-term stability of their atmospheres will be in place.

\subsection{Dedicated EUV Eruption Program}

The Dedicated EUV Eruption Program (DEEP) survey executes monitoring campaigns of 24 select stars to measure EUV flare frequency distributions and CME rates on individual, high-priority targets in the solar neighborhood. 
ESCAPE measures the EUV flare frequency distribution of carefully selected F, G, K, and M dwarf systems on temporal baselines of $\sim 20$ to 40 times longer than the best available datasets from HST. Similarly, there has only been one comparable M dwarf campaign at x-ray wavelengths ( 0.15 to $15 \mathrm{keV}$; Kowalski et al. - in prep), and these data do not sample the $10^{5-6} \mathrm{~K}$ emission that contributes the majority of the stellar EUV flux. ${ }^{88}$ Archival Kepler and TESS observations of white-light flares may also provide connections between the optical and EUV frequencies of superflares (e.g., Loyd et al. 2018b; ${ }^{46}$ Howard et al. 2020)..$^{38}$

The average CME rate of the Sun is $\sim 3.5 \mathrm{CMEday}^{-1}$ and is $\sim 10 \mathrm{CMEday}^{-1}$ at solar maximum. ${ }^{89}$ The Sun is less active than other stars of its type (e.g., Reinhold et al. 2020), ${ }^{90}$ so we conservatively estimate a CME rate of $5 \mathrm{CME} \mathrm{day}^{-1}$ for Sun-like stars. Stellar CME rates are likely well-approximated by a Poisson distribution like the Sun, so the uncertainty on the CME frequency per day is the square root of the number of CMEs observed in a monitoring campaign divided by the monitoring duration. Therefore, observing nine CMEs results in a 3- $\sigma$ constraint on the CME rate. While not every CME is preceded by a dimming event, most are. Veronig et al. find that $97 \%$ of CMEs have a corresponding irradiance dimming. However, only $\sim 50 \%$ of those will occur on the observable side of the star. Finally, approximately one-third of all solar dimming events meet ESCAPE's 3- $\sigma$ spectrophotometric detection threshold (dimming depths $\geq 6 \%$ in Fe IX $171 \AA$ and Fe X $174 \AA$ ). Combining the above numbers with ESCAPE 's DEEP survey observing efficiency (78\%), the expected detection rate for solar-like CMEs with ESCAPE is approximately 0.63 CMEs/day. ESCAPE will monitor each star in the DEEP survey for $\approx 14$ days to provide sufficient temporal baselines to observe nine CMEs/target, resulting in a 3- $\sigma$ determination of the CME rate per star, from which CME-driven atmospheric escape will be estimated.

ESCAPE's spectral coverage and spectral resolution $(\Delta \lambda<1.5 \AA)$ are designed to spectrally isolate specific emission lines for temporally resolved lightcurve analysis over a range of coronal ionization states (Fe VIII-Fe XX). ESCAPE has the sensitivity to detect solar-like dimming events (6\% max dimming depth in Fe IX $171 \AA$ and Fe X $174 \AA$ A, summed over ten 30 minute exposures) to a distance of $6 \mathrm{pc}$. ESCAPE will survey $10 \mathrm{~F}, \mathrm{G}$, and $\mathrm{K}$ stars for solar-like CMEs as part of the DEEP survey. For these $10 \mathrm{~F}, \mathrm{G}$, and $\mathrm{K}$ stars, we expect ESCAPE will detect over 90 solar-like coronal dimming events. Therefore, ESCAPE will likely characterize the frequency of stellar CMEs for the first time. Deeper dimming events or those detectable in broadband spectral binning ${ }^{71}$ are even more readily detectable in ESCAPE time-series observations. We note that while dimming events from the hot coronae associated with very active stars may be detectable in the $\mathrm{x}$-rays, EUV spectral coverage is required to sample the lower quiescent coronal emission temperatures typical of the more numerous, lower activity, planet hosting stars.

Magnetic fluxes on active M stars can be factors of 10 to 1000 times higher than global solar magnetic fluxes, ${ }^{91,92}$ potentially trapping the charged particle explosions associated with CMEs. ${ }^{93}$ Simulations by our team indicate that CMEs with energy $>10^{34}$ ergs will escape magnetic confinement from active $\mathrm{M}$ dwarfs and be detectable with ESCAPE (Jin et al. - in prep). ESCAPE's observational capabilities enable us to search for EUV dimming signatures from $\mathrm{M}$ dwarfs for the first time, providing empirical constraints on the particle deposition into the atmospheres of orbiting planets.

\section{Implementation: The ESCAPE Science Payload}

The ESCAPE science mission requires measurements of the EUV and FUV fluxes of a sample of low-mass stars. To adequately carry out this program, ESCAPE must be at least 25-times more efficient across the 100 to $500 \AA$ EUV band than any previous astrophysics spectrograph to operate in this range (EUVE DS/S; Bowyer and Malina, 1991, ${ }^{30}$ Chandra LETG/HRC-S; Brinkman et al., 2000).${ }^{94}$ ESCAPE's efficiency gain compared to previous missions is achieved with an instrument specifically designed to meet the ESCAPE objectives; i.e., optimized to be a cool star characterization instrument.

The ESCAPE telescope is the grazing incidence analog of a Gregorian [known as a HettrickBowyer (HB), Hettrick and Bowyer], ${ }^{95}$ with a $46-\mathrm{cm}$ parabolic primary mirror, a prime focus aperture (PFA), and a re-focusing elliptical secondary. An accessible prime focus is a defining

J. Astron. Telesc. Instrum. Syst. $\quad$ 014006-10 Jan-Mar 2022 • Vol. 8(1) 
feature of Gregorian telescopes, and one of the critical elements that enables the high efficiency of ESCAPE; the field-limiting PFA provides stray-light suppression without costly bandpass limiting filters or additional reflections that would be prohibitive to meeting the system throughput requirements. The telescope is provided by a joint NASA/Marshall Space Flight Center (MSFC) and Smithsonian Astrophysical Observatory (SAO) team. Figure 7 shows the ESCAPE telescope and science instrument with key components labeled.

The ESCAPE spectrograph intercepts the converging beam from the telescope with an array of etched silicon gratings supplied by Pennsylvania State University (PSU). The grating bank is divided into two channels to optimize performance over a broad EUV bandpass: the G20 (78 to $339 \AA$ ) and the G40 (94 to $564 \AA$; Table 1). The gratings are ruled with a quasi-radial groove pattern to maintain a constant linear dispersion. Each channel is divided into segments sized to be compatible with a standard 6 in. silicon wafer for ease of manufacture and heritage. Segments are co-aligned with the aid of an optical grating ruled onto a nominally unilluminated portion of the substrate.

The resulting spectra are curved in an arc-of-diffraction common in all radial groove spectrographs and recorded onto a microchannel plate (MCP) detector developed by the University of California, Berkeley (UCB) - the workhorse detector type for all EUV and FUV instruments. The zero-order reflections off of the G20 and G40 gratings are folded back onto two normal

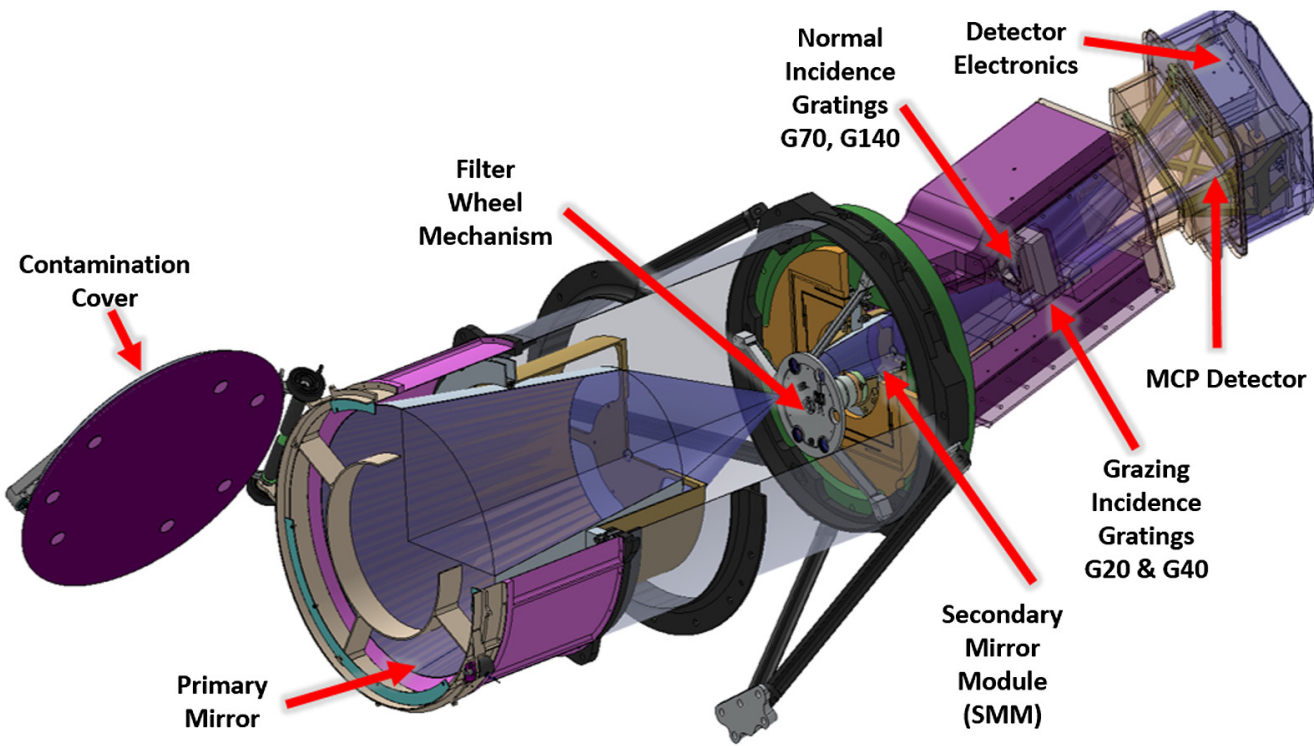

Fig. 7 A schematic representation of the ESCAPE science instrument: a grazing incidence Gregorian telescope (Sec. 6.1.1) feeding a grazing incidence spectrograph (Sec. 6.1.2). The primary mirror diameter is $0.46 \mathrm{~m}$ and the end-to-end length of the instrument is $\sim 2 \mathrm{~m}$. Major components are labeled.

Table 1 ESCAPE spectroscopic modes.

\begin{tabular}{lcccc}
\hline \hline Grating & $\begin{array}{c}\text { Bandpass } \\
(\AA)\end{array}$ & $\begin{array}{c}\text { Spatial } \\
\text { resolution }(\mu \mathrm{m})\end{array}$ & $\begin{array}{c}\text { Spectral } \\
\text { resolution }(\AA)\end{array}$ & $\begin{array}{c}\text { Peak } A_{\text {eff }} \\
\left(\mathrm{cm}^{2}\right)\end{array}$ \\
\hline G20 & 78 to 339 & 500 & $0.91^{\mathrm{a}}$ & 67.4 \\
G40 & 94 to 564 & 500 & $0.65^{\mathrm{a}}$ & 35.2 \\
G70 & 600 to 825 & 475 & $3.6^{\mathrm{b}}$ & 2.6 \\
G140 & 1250 to 1650 & 475 & $6.1^{\mathrm{b}}$ & 4.5 \\
\hline \hline
\end{tabular}

aEvaluated at $171 \AA$.

${ }^{\mathrm{b}}$ Average resolution over the band. 
incidence re-focusing gratings, the G70 (600 to $825 \AA$ ) and G140 (1250 to $1650 \AA$ ), to obtain long-wave EUV and FUV coverage. These spectra are recorded onto the same MCP detector as the grazing channels, providing this spectral coverage with minimal additional complexity. The ESCAPE instrument covers the required broad spectral range simultaneously with a fixed optical configuration that requires no mechanisms or bandpass limiting optics during normal science operations.

\subsection{Instrument Subsystems}

In the following sections, we describe the key components of the ESCAPE instrument in greater detail.

\subsubsection{ESCAPE telescope}

The ESCAPE telescope is a grazing incidence Gregorian with a prime focus point in between the primary and secondary mirrors (see Fig. 7; Hettrick and Bowyer). ${ }^{95}$ The final architecture was chosen after a series of trade studies to maximize efficiency and minimize complexity.

The ESCAPE parabolic primary mirror is a $1-\mathrm{mm}$ thick $\times 500-\mathrm{mm}$ long, $46-\mathrm{cm}$ maximum diameter nickel shell with a focus point at the PFA pinhole (500 $\mu$ m diameter). The PFA restricts the field-of-view of ESCAPE to a top-hat function with a 1.6 arcmin full-transmission zone and wings extending out to a 3.6-arcmin diameter. This restricted field-of-view limits geocoronal airglow in the system without requiring costly bandpass limiting transmission filters. The diverging beam is re-focused by a $1-\mathrm{mm}$ thick $\times 152-\mathrm{mm}$ long, $13-\mathrm{cm}$ outer diameter elliptical secondary mirror.

The mirror shells will be fashioned at NASA/MSFC by electro-forming a nickel-cobalt alloy onto a super-polished aluminum mandrel figured to the desired optic prescription. This is known as electro-formed nickel replication, the same mirror technology used for IXPE, XMM-Newton, FOXSI, MaGIXS, and other NASA flight programs. Both telescope optics are coated with $20 \mathrm{~nm}$ of zirconium in the coating facilities at SAO (Romaine et al., 2011). ${ }^{96}$ Zirconium has excellent performance at critical ESCAPE wavelengths and is widely used for thin film applications.

\subsubsection{ESCAPE spectrograph}

Grazing Incidence Gratings-the ESCAPE short-wavelength EUV channels utilize quasi-radial off-plane blazed gratings at grazing incidence. ${ }^{97,98}$ Each channel intercepts roughly half of the telescope power at average graze angles of $\theta=11 \mathrm{deg}(\mathrm{G} 20)$ and $19 \mathrm{deg}(\mathrm{G} 40)$. The groove patterns are angled to match the converging telescope rays to maintain a constant linear dispersion, increasing the groove density as the rays approach focus. Light is diffracted along an arc of diffraction, with spectra offset in the cross-dispersion direction. The bandpass of each channel is extended by leveraging multiple spectral orders. Higher spectral order contributions are suppressed by the telescope graze angles; all $m \geq 3$ order in G20 and $m \geq 4$ in G40 have effective area $\left(A_{\text {eff }}\right)<0.5 \mathrm{~cm}^{2}$.

The gratings are lithographically ruled into single crystal silicon wafers following a process developed by PSU under a NASA Strategic Astrophysics Technology (SAT) award ${ }^{99}$ and demonstrated with ESCAPE grating prescriptions. ${ }^{100}$ The wafers are cut from the parent boule so the $\langle 111\rangle$ crystal plane orientation matches the desired blaze angles. This grating fabrication technique produces smooth and uniform blaze facets, maximizing diffraction efficiency while readily meeting the low scatter requirement of $I / I_{o}<10^{-3}$ at $10 \AA$ from an emission line to suppress scattered H I Ly $\alpha$. The segments are diced to a rectangular form and bonded to kovar mounts with $X, Y$, and $\theta$ adjustment capability to aid in segment alignment.

Normal Incidence Gratings - The NI gratings are supplied by Horiba Jobin-Yvon; the normal incidence channels leverage unused detector area to provide essential science capability with minimal added complexity. The G70 and G140 channels consist of 14-mm square flat fold mirrors that pickoff the reflected zero-order light from the G40 and G20, respectively, and direct it to concave re-focusing holographic gratings. The spectra are imaged onto the same detector as the grazing incidence channels. The G140 channel is coated in $\mathrm{MgF}_{2}$ protected aluminum, whereas

J. Astron. Telesc. Instrum. Syst. 014006-12 Jan-Mar 2022 • Vol. 8(1) 
the G70 channel is coated in silicon carbide ( $\mathrm{SiC}$ ). The gratings are bonded to an aluminum support structure with manual tip/tilt adjustment for alignment. The fold mirrors are mounted on the detector support truss in a small enclosure to baffle stray light. A 4-mm thick, 6-mm diameter $\mathrm{CaF}_{2}$ window intercepts the G140 beam to attenuate $>95 \%$ of geocoronal H I Ly $\alpha$.

\subsubsection{ESCAPE detector system}

The ESCAPE spectra are imaged by a $125 \times 40 \mathrm{~mm}$ MCP detector system with mild focal plane curvature $(0.5 \mathrm{~m})$ and a cross delay line (XDL) readout anode. The baseline design uses borosilicate-glass MCPs activated by atomic layer deposition, which have flight heritage on large formats and with curvatures (DEUCE; Erickson et al., 2021, ${ }^{101}$ SISTINE; Cruz-Aguirre et al., 2021: ${ }^{102}$ JUNO-UVS; Davis et al., 2018). ${ }^{103}$

UV light sensing is accomplished with an opaque $\mathrm{KBr}$ photocathode; a standard option for EUV and FUV missions (including EUVE and FUSE; Vallerga et al., Sahnow et al.). ${ }^{104,105}$ Consistent with previous flight detectors, we employ a charged $95 \%$ transmission grid to enhance the efficiency by collecting events that impinge on the detector face. The structure of this grid has been examined as part of the ESCAPE ray trace analysis and has been observed to have no noticeable impact on the PSF. The XDL readout anode is based on the SISTINE and DEUCE multilayer designs (France et al. 2016 ${ }^{106}$ and Erickson et al. 2021). ${ }^{101}$

ESCAPE utilizes an electronics package with flight heritage on numerous missions, including ICON-EUV, JUNO-UVS, JUICE-UVS, EUROPA-UVS, and EMM-EMUS. ${ }^{107-110}$ This package utilizes low-power amplifiers/discriminators for the start and stop signals of each axis of the XDL, followed by time to digital conversion, and an FPGA (ACTEL AX/RT family) to convert the raw event time of arrival differences into $x$ and $y$ and signal amplitudes outputted as 32-bit LVDS (13-bit X, 12-bit Y, and 7-bit pulse height). Maximum global count rates for primary science targets, including airglow contributions, are $<1000 \mathrm{~Hz}$, posing no counting rate challenges for the detector system.

\subsection{Instrument Performance}

The system effective area is a function of the reflection efficiency of the optics $(R)$, efficiency of the gratings $\left(\epsilon_{g}\right)$, and quantum efficiency of the detector, multiplied by the geometric collecting area of the telescope $\left(\mathrm{A}_{\mathrm{geo}}\right)$ :

$$
A_{\text {eff }}(\lambda)=A_{\text {geo }} R(\lambda, \theta) \epsilon_{g}(\lambda) D Q E(\lambda) .
$$

The reflectivity of each optic is a function of the wavelength and graze angle, $\theta$, which varies across the surface. To calculate this function, a grid of rays is traced through the system, with the graze angle at each optic intercept point used to calculate the power reflected as a function of wavelength. The rays are evenly spaced at the aperture to properly power-weight this function.

The total $A_{\text {eff }}$ of ESCAPE (Fig. 8) is the sum of all diffraction orders, as well as the sum of the two GI channels for where the wavelength range overlaps. The $A_{\text {eff }}$ of the NI channels (G70 and $\mathrm{G140}$ ) is calculated with added terms for the fold mirror, $\mathrm{CaF}_{2}$ filter (G140), and zero order reflection off of the corresponding GI grating.

The point-spread-function (PSF) is the sum of contributions from the intrinsic grating distortion, optical mount stresses, 1-sigma pointing jitter, maximum allowable mirror fabrication errors and maximum allowable detector resolution. This PSF produces a predicted RMS spectral resolution performance at $171 \AA$ for the grazing incidence channels of $0.91 \AA$ in $\mathrm{G} 20$ and $0.66 \AA$ in G40 (second order), and normal incidence performance of $3.6 \AA$ in G70 and $6.1 \AA$ in G140 (see Table 1), averaged over the normal incidence mode bandpasses. The raytraced PSF spot for the G20 at $171 \AA$ is shown in Fig. 9.

\subsection{Simulated Data Example}

We carried out science performance calculations using a conservative spot size corresponding to the baseline requirement of $1.5 \AA$ spectral resolution at $171 \AA$, lower than the projected

J. Astron. Telesc. Instrum. Syst. $\quad$ 014006-13 Jan-Mar 2022 • Vol. 8(1) 
France et al.: Extreme-ultraviolet Stellar characterization for atmospheric physics and evolution mission...

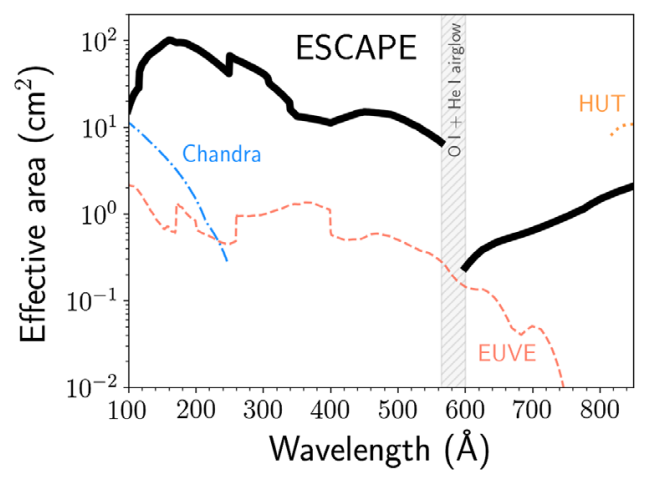

Fig. 8 A comparison of the ESCAPE effective area (solid black line) compared with previous astrophysics missions covering the 100 to $912 \AA$ EUV bandpass. ESCAPE intentionally introduces a gap in spectral coverage between $\sim 550$ and $600 \AA$ to avoid strong O II and He I geocoronal emission.

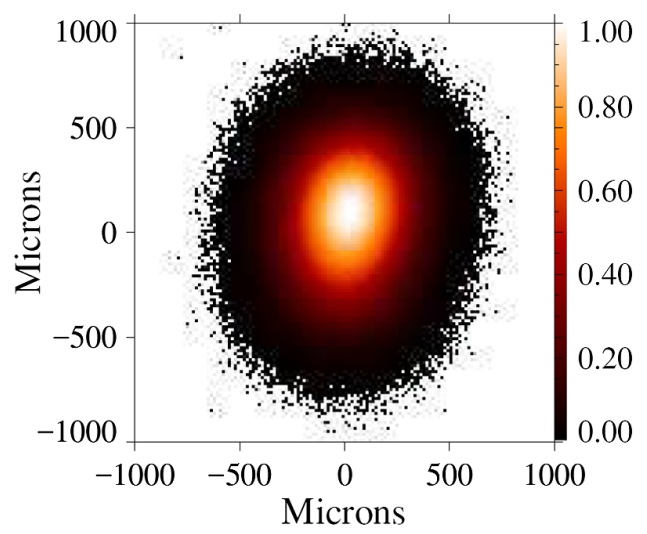

Fig. 9 Projected PSF for the G20 mode at $171 \AA$, sampled at the detector digital pixel scale $(\sim 14 \mu \mathrm{m} \times 14 \mu \mathrm{m})$.

instrument performance. A simulated two-dimensional detector image of a SEEN survey target is shown in Fig. 10(a). ESCAPE's targets are cool stars, dominated by coronal and chromospheric line emission. Therefore, the order overlap associated with continuum sources that was an issue for EUVE is mitigated for ESCAPE and wavelength identification can be accomplished without wavelength blocking filters. For ESCAPE, the wavelength definition can be cleanly inferred from comparison of one-dimensional (1D) spectral extractions with solar or synthetic stellar spectra (as illustrated by the minimal spectral order overlap for solar-type stars shown in Fig. 10, center panels). More sophisticated forward modeling techniques, used on grating spectra at X-ray wavelengths (see, e.g., Brinkman et al.), ${ }^{94}$ will be developed to identify spectral features by their location in the two-dimensional (2D) spectrogram.

Spectral data are order-sorted by three methods: (1) laboratory spectra obtained during instrument integration and testing provide a map of spectral detector locations as a function of input wavelength for known calibration gases, (2) on-orbit checkout calibration observations of cool stars with EUVE observations will be used to validate forward modeling analyses of coronal models; pre-flight simulations show clear delineation of different wavelengths in 1D spectral traces for G20 and G40 channels (Fig. 10, center), and (3) order-sorting filters (manufactured by Luxel) isolating short-, medium-, and long-wavelength EUV bands are wellestablished in solar missions and will be used to validate the forward modeling order sorting analysis as well as reject soft x-ray continuum leaking into the ESCAPE band for stars with high-temperature coronae.

An $\mathrm{Al}+\mathrm{Mg}$ filter isolates the first order G40 spectrum (and supports the calculation of a predictive DEM model to guide the extraction). The G40 Open - Al+Mg difference spectra are 


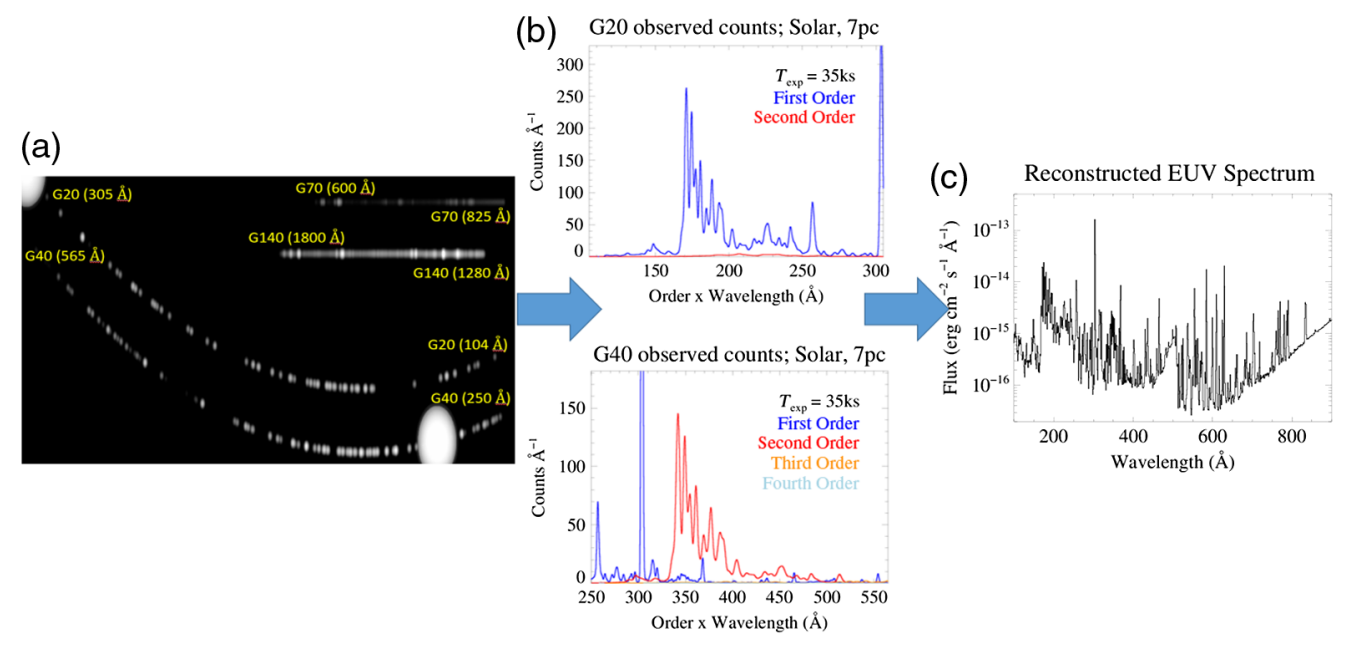

Fig. 10 A schematic representation of the ESCAPE science data flow for an inactive solar type star at a distance of $7 \mathrm{pc}$, with a typical SEEN exposure time of $35 \mathrm{ksec}$. (a) Level 3a science products contain the $(\lambda, y, t)$ photon lists, including order identifications. The oval features are He II $304 \AA$ airglow, which are spectrally isolated on the detector and do not impact the majority of the science spectrum. (b) 1D spectra are extracted and different wavelengths are readily identified in the G20 and G40 channels. (c) Order-sorted 1D spectra are combined with interstellar attenuation corrections and differential emission measure models to produce full extreme ultraviolet irradiance spectra.

cross-correlated with the $\mathrm{Zr}+\mathrm{MoSi}_{2} \mathrm{G} 20$ spectra to isolate the first and second order G20 and G40 spectra. An indium filter tests for short-wavelength bremsstrahlung leaking into the ESCAPE band. Filters are only used for initial wavelength/order calibration activities; an open aperture position is used for all SEEN and DEEP survey observations.

Figure 10 shows the typical data flow for ESCAPE observations. The Level 2 products contain the instrument data organized by observations, photon lists at full detector resolution, with all of the detector related corrections applied (application of ground-based flat-field, thermal and geometric distortion maps, and 2D walk correction as necessary; e.g., COS Data Handbook 2015; France et al. 2011 ${ }^{111}$ ). The photon-list data are time-tagged to $100 \mathrm{msec}$ accuracy.

Two Level 3 (L3) data products are generated: The L3a products preserve the full time-tag photon list from Level 2, with the addition of corresponding grating order probability (the fraction contributed by each order to the best-fit forward model spectrum at a given detector position) and wavelength for the four expected orders. Wavelength solutions are provided to the end-user for the creation of lightcurves in individual spectral features (as is done for HSTCOS). Level 3a science products contain the $(\lambda, y$, and $t)$ photon lists that are required for stellar flare and CME characterization [Fig. 10(a)]. L3b contain the fully calibrated, one dimensional spectrum in ergs cm ${ }^{-2} \mathrm{~s}^{-1} \AA^{-1}$ as a function of wavelength (in $\AA$ ) for each observation (both in individual orders and a final coadded spectrum). L3b data are combined with interstellar attenuation corrections and a DEM model to produce full extreme ultraviolet irradiance spectra [Fig. 10(b)]. The ESCAPE data will be hosted at the Mikulski Archive for Space Telescopes (MAST) archive.

Level 3a and 3b data products will be produced for individual exposures and exposure time integrated for each ESCAPE target to facilitate dissemination and use by the astronomical community. Level $3 \mathrm{~b}$ science products contain the full 1D EUV and FUV stellar spectra that are required to measure and reconstruct the EUV irradiance incident on orbiting planets. Figure 4 shows a simulated SEEN observation (extracted counts spectrum) of the nearby planet-hosting M dwarf Proxima Cen, compared to an archival EUVE spectrum of the same source. Figure 11 shows a Level $3 b$ data product of a simulated DEEP observation (the flux spectrum) of Proxima Cen and the predicted signal-to-noise ratio as a function of wavelength that includes the influence of local ISM absorption on the ESCAPE observations. 
(a) Proxima centauri at $1.3 \mathrm{pc}$

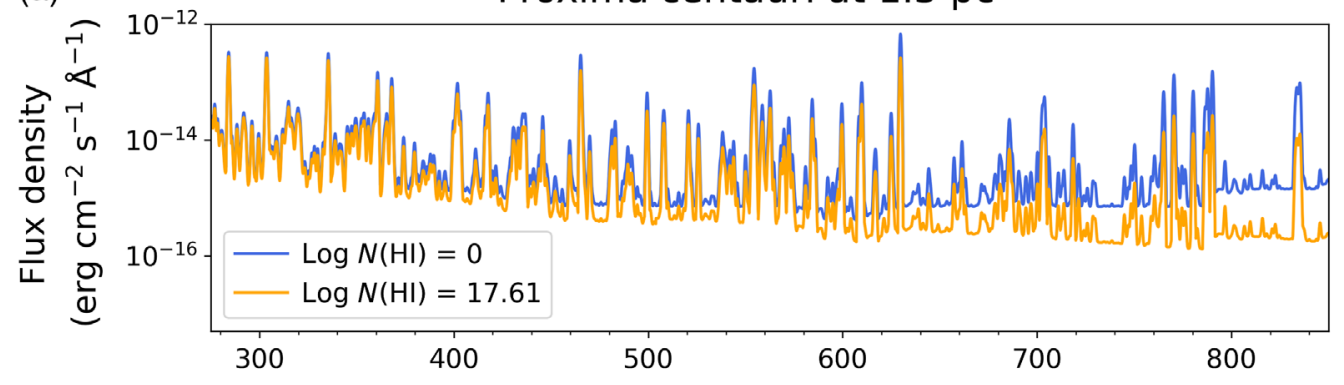

(b)

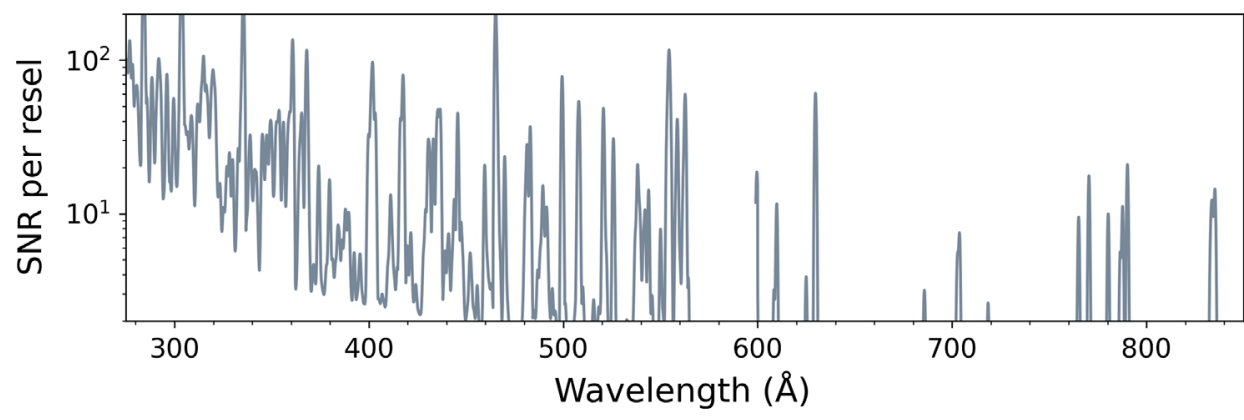

Fig. 11 (a) Simulated observation of the full EUV spectrum of Proxima Cen comparing the intrinsic (blue) and ISM-absorbed ESCAPE data (orange). (b) Signal-to-noise per $1.5 \AA$ Å resolution element of a DEEP Survey observation of Proxima Cen.

\section{ESCAPE Development Timeline}

ESCAPE completed an initial Phase A study in mid-2021 but was not selected for full implementation. ESCAPE will be re-proposed to the next SMEX opportunity, presumably in 2024. If ESCAPE is selected in that round, Phase B would begin in late 2025, culminating in the instrument and mission level preliminary design reviews in late 2026, respectively. Phase C includes comprehensive design review in late Q2 2027 and extends through August 2028. Phase D would run through the August 2029 launch and on-orbit commissioning. On-orbit spectral resolution tests are made by observing the unresolved and spectrally isolated Fe XIV and Fe XVI lines as these spectral diagnostics are expected to be present in all cool star observations (coronal line widths are $<10 \%$ of instrumental resolution). Effective area as a function of wavelength is calibrated in flight by performing observations of well-studied hot white dwarf calibration stars. Phase E comprises the 20 month science mission when the full SEEN and DEEP surveys are executed; ESCAPE maintains a $26 \%$ mission lifetime margin to complete the baseline science mission.

\section{Acknowledgments}

The ESCAPE Phase A mission was managed at the Laboratory for Atmospheric and Space Physics at the University of Colorado Boulder under NASA contract No. 80GSFC21C0003. Portions of this manuscript appeared as SPIE proceeding 11821-001. The ESCAPE team acknowledges the numerous invaluable discussions with colleagues on the need for and approach to an EUV spectroscopy mission over the previous five years. In particular, KF thanks Alex Brown, Parke Loyd, and Tom Woods for enjoyable conversations on EUV spectroscopy and variability.

\section{References}

1. C. P. Johnstone et al., "The evolution of stellar rotation and the hydrogen atmospheres of habitable-zone terrestrial planets," Astrophys. J. Lett. 815, L12 (2015). 
2. C. P. Johnstone, M. Bartel, and M. Güdel, "The active lives of stars: a complete description of the rotation and XUV evolution of F, G, K, and M dwarfs," Astron. Astrophys. 649, A96 (2021).

3. L. Tu et al., "The extreme ultraviolet and x-ray Sun in time: high-energy evolutionary tracks of a solar-like star," Astron. Astrophys. 577, L3 (2015).

4. U. V. Amerstorfer et al., "Escape and evolution of Mars's CO atmosphere: influence of suprathermal atoms," J. Geophys. Res. (Planets) 122, 1321-1337 (2017).

5. A. Vidal-Madjar et al., "Detection of oxygen and carbon in the hydrodynamically escaping atmosphere of the extrasolar planet HD 209458b," Astrophys. J. Lett. 604, L69-L72 (2004).

6. J. L. Linsky et al., "Observations of mass loss from the transiting exoplanet HD 209458b," Astrophys. J. 717, 1291-1299 (2010).

7. G. E. Ballester and L. Ben-Jaffel, "Re-visit of HST FUV observations of the hot-Jupiter system HD 209458: No Si III detection and the need for COS transit observations," Astrophys. J. 804, 116 (2015).

8. L. Fossati et al., "Metals in the exosphere of the highly irradiated planet WASP-12b," Astrophys. J. Lett. 714, L222-L227 (2010).

9. C. A. Haswell et al., "Near-UV absorption, chromospheric activity, and star-planet interactions in the WASP-12 system," Astrophys. J. 760, 79 (2012).

10. D. K. Sing et al., "The Hubble Space Telescope PanCET program: exospheric Mg II and Fe II in the near-ultraviolet transmission spectrum of WASP-121b using jitter decorrelation," Astron. J. 158, 91 (2019).

11. P. E. Cubillos et al., "Near-ultraviolet transmission spectroscopy of HD 209458b: evidence of ionized iron beyond the planetary roche lobe," Astron. J. 159, 111 (2020).

12. Y. N. Kulikov et al., "Atmospheric and water loss from early Venus," PLANSS 54(13-14), 1425-1444 (2006).

13. H. I. M. Lichtenegger et al., "Solar XUV and ENA-driven water loss from early Venus' steam atmosphere," J. Geophys. Res. (Space Phys.) 121, 4718-4732 (2016).

14. C. Dong et al., "Is proxima centauri b habitable? A study of atmospheric loss," Astrophys. J. Lett. 837, L26 (2017).

15. V. S. Airapetian et al., "Impact of space weather on climate and habitability of terrestrialtype exoplanets," Int. J. Astrobiol. 19, 136-194 (2020).

16. G. Gronoff et al., "Atmospheric escape processes and planetary atmospheric evolution," J. Geophys. Res.: Space Phys. 125(8), e2019JA027639 (2020).

17. R. M. Ramirez and L. Kaltenegger, "The habitable zones of pre-main-sequence stars," Astrophys. J. Lett. 797, L25 (2014).

18. R. Luger and R. Barnes, "Extreme water loss and abiotic $\mathrm{O}_{2}$ buildup on planets throughout the habitable zones of M Dwarfs," Astrobiology 15, 119-143 (2015).

19. R. D. Wordsworth, L. K. Schaefer, and R. A. Fischer, "Redox evolution via gravitational differentiation on low-mass planets: implications for abiotic oxygen, water loss, and habitability," Astron. J. 155, 195 (2018).

20. T. N. Woods et al., "Solar irradiance reference spectra (SIRS) for the 2008 whole heliosphere interval (WHI)," Geophys. Res. Lett. 36, 1101 (2009).

21. J. M. Fontenla et al., "Semi-empirical modeling of the photosphere, chromosphere, transition region, and corona of the M-dwarf host Star GJ 832," Astrophys. J. 830, 154 (2016).

22. K. France et al., "The MUSCLES treasury survey. I. Motivation and overview," Astrophys. J. 820, 89 (2016).

23. R. O. P. Loyd et al., "The muscles treasury survey. III. X-ray to infrared spectra of $11 \mathrm{~m}$ and k stars hosting planets," Astrophys. J. 824(2) (2016).

24. J. J. Drake et al., "Pointing Chandra toward the extreme ultraviolet fluxes of very low Mass stars," Astrophys. J. 893, 137 (2020).

25. J. L. Linsky, J. Fontenla, and K. France, "The intrinsic extreme ultraviolet fluxes of F5 V TO M5 V stars," Astrophys. J. 780, 61 (2014).

26. J. Sanz-Forcada et al., "Estimation of the XUV radiation onto close planets and their evaporation," Astron. Astrophys. 532, A6 (2011). 
France et al.: Extreme-ultraviolet Stellar characterization for atmospheric physics and evolution mission...

27. I. Ribas et al., "The full spectral radiative properties of Proxima Centauri," $A \& A \mathbf{6 0 3}$ (2017).

28. K. Garcia-Sage et al., "On the magnetic protection of the atmosphere of proxima centauri b," Astrophys. J. Lett. 844, L13 (2017).

29. V. S. Airapetian et al., "How hospitable are space weather affected habitable zones? The role of ion escape," Astrophys. J. Lett. 836, L3 (2017).

30. S. Bowyer and R. F. Malina, "The extreme ultraviolet explorer mission," Adv. Space Res. 11, 205-215 (1991).

31. J. J. Drake, J. M. Laming, and K. G. Widing, "Stellar coronal abundances. II. The first ionization potential effect and its absence in the corona of procyon," Astrophys. J. 443, 393 (1995).

32. J. J. Drake, J. M. Laming, and K. G. Widing, "Stellar coronal abundances. V. Evidence for the first ionization potential effect in $\alpha$ Centauri," Astrophys. J. 478, 403-416 (1997).

33. L. M. Walkowicz and S. L. Hawley, "Tracers of chromospheric structure. I. Observations of Ca II K and Ho in M Dwarfs," Astron. J. 137, 3297-3313 (2009).

34. T. N. Woods et al., "Extreme ultraviolet variability experiment (EVE) on the solar dynamics observatory (SDO): overview of science objectives, instrument design, data products, and model developments," Solar Phys. 275, 115-143 (2012).

35. A. Segura et al., "The effect of a strong stellar flare on the atmospheric chemistry of an Earth-like planet orbiting an M Dwarf," Astrobiology 10, 751-771 (2010).

36. J. R. A. Davenport, "The Kepler catalog of stellar flares," Astrophys. J. 829, 23 (2016).

37. M. N. Günther et al., "Stellar flares from the first TESS data release: exploring a new sample of M Dwarfs," Astron. J. 159, 60 (2020).

38. W. S. Howard et al., "EvryFlare. III. Temperature evolution and habitability impacts of dozens of superflares observed simultaneously by evryscope and TESS," Astrophys. J. 902, 115 (2020).

39. S. L. Hawley and B. R. Pettersen, "The great flare of 1985 April 12 on AD Leonis," Astrophys. J. 378, 725-741 (1991).

40. O. Venot et al., "Influence of Stellar flares on the chemical composition of exoplanets and spectra," Astrophys. J. 830, 77 (2016).

41. M. Audard et al., "Extreme-ultraviolet flare activity in late-type Stars," Astrophys. J. 541, 396-409 (2000).

42. K. France et al., "The high-energy radiation environment around a 10 Gyr M Dwarf: habitable at last?" Astron. J. 160, 237 (2020).

43. B. W. Jones and P. N. Sleep, "Habitability of exoplanetary systems with planets observed in transit," Mon. Not. R. Astron. Soc. 407, 1259-1267 (2010).

44. S. L. Hawley et al., "Multiwavelength observations of flares on AD leonis," Astrophys. J. 597, 535-554 (2003).

45. R. O. P. Loyd and K. France, "Fluctuations and flares in the ultraviolet line emission of cool stars: implications for exoplanet transit observations," Astrophys. J. Suppl. Ser. 211, 9 (2014).

46. R. O. P. Loyd et al., "The MUSCLES treasury survey. V. FUV Flares on active and inactive M Dwarfs," Astrophys. J. 867, 71 (2018).

47. A. A. West et al., "An activity-rotation relationship and kinematic analysis of nearby mid-to-late-type M Dwarfs," Astrophys. J. 812, 3 (2015).

48. M. L. Khodachenko et al., "Coronal mass ejection (CME) activity of low mass M Stars as an important factor for the habitability of terrestrial exoplanets. I. CME Impact on expected magnetospheres of Earth-like exoplanets in close-in habitable zones," Astrobiology 7, 167-184 (2007).

49. H. Lammer et al., "Coronal mass ejection (CME) activity of low mass $m$ stars as an important factor for the habitability of terrestrial exoplanets. II. CME-induced ion pick up of Earth-like exoplanets in close-in habitable zones," Astrobiology 7, 185-207 (2007).

50. A. Cherenkov et al., "The influence of coronal mass ejections on the mass-loss rates of hot-Jupiters," Astrophys. J. 846, 31 (2017).

51. M. A. Tilley et al., "Modeling repeated M Dwarf flaring at an Earth-like planet in the habitable zone: atmospheric effects for an unmagnetized planet," Astrobiology 19, 64-86 (2019). 
52. E. Chassefière, "Hydrodynamic escape of oxygen from primitive atmospheres: applications to the cases of Venus and Mars," Icarus 124, 537-552 (1996).

53. B. M. Jakosky et al., "MAVEN observations of the response of Mars to an interplanetary coronal mass ejection," Science 350, 643 (2015).

54. B. M. Jakosky et al., "Loss of the Martian atmosphere to space: present-day loss rates determined from MAVEN observations and integrated loss through time," Icarus $\mathbf{3 1 5}$, 146-157 (2018).

55. S. Yashiro et al., "Different power-law indices in the frequency distributions of flares with and without coronal mass ejections," Astrophys. J. Lett. 650, L143-L146 (2006).

56. R. A. Osten and S. J. Wolk, "Connecting flares and transient mass-loss events in magnetically active stars," Astrophys. J. 809, 79 (2015).

57. P. Odert et al., "Stellar coronal mass ejections-I. Estimating occurrence frequencies and mass-loss rates," Mon. Not. R. Astron. Soc. 472, 876-890 (2017).

58. M. K. Crosley and R. A. Osten, "Constraining Stellar coronal mass ejections through multi-wavelength analysis of the active M Dwarf EQ peg," Astrophys. J. 856, 39 (2018).

59. B. M. Haisch et al., "Coordinated Einstein and IUE observations of a disparitions brusques type flare event and quiescent emission from Proxima Centauri.," Astrophys. J. 267, 280-290 (1983).

60. S.-P. Moschou et al., "A monster CME obscuring a demon star flare," Astrophys. J. 850, 191 (2017).

61. L. K. Harra et al., "The characteristics of solar X-class flares and CMEs: a paradigm for stellar superflares and eruptions?" Solar Phys. 291, 1761-1782 (2016).

62. A. C. Sterling and H. S. Hudson, "Yohkoh SXT observations of X-ray 'dimming' associated with a halo coronal mass ejection," Astrophys. J. Lett. 491, L55-L58 (1997).

63. K. Dissauer et al., "On the detection of coronal dimmings and the extraction of their characteristic properties," Astrophys. J. 855, 137 (2018).

64. K. Dissauer et al., "Statistics of coronal dimmings associated with coronal mass ejections. I. Characteristic dimming properties and flare association," Astrophys. J. 863, 169 (2018).

65. M. J. Aschwanden et al., "First measurements of the mass of coronal mass ejections from the EUV dimming observed with STEREO EUVI A+B spacecraft," Astrophys. J. 706, 376-392 (2009).

66. A. A. Reinard and D. A. Biesecker, "The relationship between coronal dimming and coronal mass ejection properties," Astrophys. J. 705, 914-919 (2009).

67. J. P. Mason et al., "Mechanisms and observations of coronal dimming for the 2010 August 7 event," Astrophys. J. 789, 61 (2014).

68. J. P. Mason et al., "Relationship of EUV irradiance coronal dimming slope and depth to coronal mass ejection speed and mass," Astrophys. J. 830, 20 (2016).

69. J. P. Mason et al., "The SDO/EVE solar irradiance coronal dimming index catalog. I. Methods and algorithms," Astrophys. J. Suppl. Ser. 244, 13 (2019).

70. T. N. Woods et al., "New solar extreme-ultraviolet irradiance observations during flares," Astrophys. J. 739, 59 (2011).

71. A. M. Veronig et al., "Indications of stellar coronal mass ejections through coronal dimmings," Nat. Astron. 5, 697-706 (2021).

72. N. Lehner et al., "Far ultraviolet spectroscopic explorer survey of the local interstellar medium within 200 parsecs," Astrophys. J. 595, 858-879 (2003).

73. S. Redfield and J. L. Linsky, "The structure of the local interstellar medium. IV. Dynamics, morphology, physical properties, and implications of cloud-cloud interactions," Astrophys. J. 673, 283-314 (2008).

74. B. E. Wood et al., "Stellar Ly $\alpha$ emission lines in the hubble space telescope archive: intrinsic line fluxes and absorption from the heliosphere and astrospheres," Astrophys. J. Suppl. Ser. 159, 118-140 (2005).

75. A. Youngblood et al., "The MUSCLES treasury survey ii: intrinsic lyman alpha and extreme ultraviolet spectra of $\mathrm{k}$ and $\mathrm{m}$ dwarfs with exoplanets," Astrophys. J. 824(2), 101 (2016).

76. B. E. Wood et al., "New observational constraints on the winds of M dwarf stars," Astrophys. J. 915, 37 (2021). 
France et al.: Extreme-ultraviolet Stellar characterization for atmospheric physics and evolution mission...

77. LUVOIR Final Report, "LUVOIR: Telling the Story of Life in the Universe," NASA (2019).

78. G. M. Duvvuri et al., "Reconstructing the extreme ultraviolet emission of cool dwarfs using differential emission measure polynomials," Astrophys. J. 913, 40 (2021).

79. R. O. P. Loyd et al., "HAZMAT. IV. Flares and superflares on young M Stars in the far ultraviolet," Astrophys. J. 867, 70 (2018).

80. K. France et al., "Far-ultraviolet activity levels of F, G, K, and M Dwarf exoplanet host stars," Astrophys. J. Suppl. Ser. 239, 16 (2018).

81. T. R. Ayres, "The flare-ona of EK draconis," Astron. J. 150, 7 (2015).

82. I. Ribas et al., "The habitability of Proxima Centauri b. I. Irradiation, rotation and volatile inventory from formation to the present," Astron. Astrophys. 596, A111 (2016).

83. J. S. Pineda, A. Youngblood, and K. France, "The far ultraviolet M-dwarf evolution survey. I. The rotational evolution of high-energy emissions," Astrophys. J. 911, 111 (2021).

84. A. C. Schneider and E. L. Shkolnik, "HAZMAT. III. The UV evolution of mid- to late-M Stars with GALEX," Astron. J. 155, 122 (2018).

85. C. D. Dressing and D. Charbonneau, "The occurrence of potentially habitable planets orbiting M Dwarfs estimated from the full kepler dataset and an empirical measurement of the detection sensitivity," Astrophys. J. 807, 45 (2015).

86. C. C. Stark et al., "ExoEarth yield landscape for future direct imaging space telescopes," J. Astron. Telesc. Instrum. Syst. 5, 024009 (2019).

87. R. K. Kopparapu, "A revised estimate of the occurrence rate of terrestrial planets in the habitable zones around Kepler M-dwarfs," Astrophys. J. Lett. 767, L8 (2013).

88. D. Tilipman et al., "Semiempirical modeling of the atmospheres of the M Dwarf exoplanet hosts GJ 832 and GJ 581," Astrophys. J. 909, 61 (2021).

89. N. Gopalswamy et al., "The SOHO/LASCO CME catalog," Earth Moon Planets 104, 295-313 (2009).

90. T. Reinhold et al., "The Sun is less active than other solar-like stars," Science 368(6490), 518-521 (2017).

91. J.-F. Donati et al., "The large-scale axisymmetric magnetic topology of a very-low-mass fully convective star," Science 311, 633-635 (2006).

92. D. Shulyak et al., "Strong dipole magnetic fields in fast rotating fully convective stars," Nat. Astron. 1, 0184 (2017).

93. J. D. Alvarado-Gómez et al., "Suppression of coronal mass ejections in active stars by an overlying large-scale magnetic field: a numerical study," Astrophys. J. 862, 93 (2018).

94. B. C. Brinkman et al., "Description and performance of the low-energy transmission grating spectrometer on board Chandra," Proc. SPIE 4012, 81-90 (2000).

95. M. C. Hettrick and S. Bowyer, "Grazing incidence telescopes: a new class for soft X-ray and EUV spectroscopy.," Appl. Opt. 23, 3732-3735 (1984).

96. S. Romaine et al., "Improved release coatings for electroformed x-ray optics," Proc. SPIE 8147, 81470W (2011).

97. J. Cash and C. Webster, "X-ray spectrographs using radial groove gratings," Appl. Opt. 22, 3971-3976 (1983).

98. R. L. McEntaffer, "Reflection grating spectrographs for astrophysical missions: rockets, explorers, probes, and flagship," in The Space Astrophys. Landsc. for the 2020s and Beyond, Vol. 2135, p. 5039 (2019).

99. D. M. Miles et al., "Fabrication and diffraction efficiency of a large-format, replicated X-ray reflection grating," Astrophys. J. 869, 95 (2018).

100. F. Grisé et al., "Opening the road to custom astronomical UV gratings," in Am. Astron. Soc. Meeting Abstr., Vol. 235, p. 373.16 (2020).

101. N. Erickson et al., "DEUCE: a sounding-rocket ultraviolet spectrograph for flux-calibrated B star observations across the Lyman limit," J. Astron. Telesc. Instrum. Syst. 7, 015002 (2021).

102. F. Cruz-Aguirre et al., "The assembly, calibration, and predicted performance of the SISTINE-2 sounding rocket payload," Proc. SPIE 11821, 118210H (2021).

103. M. W. Davis et al., "Stray and scattered light properties of the Juno ultraviolet spectrograph," Proc. SPIE 10699, 106990J (2018). 
France et al.: Extreme-ultraviolet Stellar characterization for atmospheric physics and evolution mission...

104. J. V. Vallerga et al., "Long-term orbital performance of the microchannel plate (MCP) detectors aboard the Extreme Ultraviolet Explorer," Proc. SPIE 2280, 57-68 (1994).

105. D. J. Sahnow et al., "On-orbit performance of the far ultraviolet spectroscopic explorer satellite," Astrophys. J. Lett. 538, L7-L11 (2000).

106. K. France et al., "The SLICE, CHESS, and SISTINE ultraviolet spectrographs: rocketborne instrumentation supporting future astrophysics missions," J. Astron. Instrum. 5(1), 1640001 (2016).

107. N. T. Darling et al., "Performance results of the ICON FUV sealed tube converters," Proc. SPIE 9601, 96010T (2015).

108. M. W. Davis et al., "Radiometric performance results of the Juno ultraviolet spectrograph (Juno-UVS)," Proc. SPIE 8146, 814604 (2011).

109. M. W. Davis et al., "Bench and thermal vacuum testing of the JUICE-UVS microchannel plate detector system," Proc. SPIE 11118, 111180Q (2019).

110. K. D. Retherford et al., "The far-UV Albedo of the Moon as a probe of the lunar cryosphere: LRO Lyman Alpha Mapping Project (LAMP) latest results," in Lunar and Planet. Sci. Conf., p. 2213 (2015).

111. K. France et al., "HST-COS observations of hydrogen, helium, carbon, and nitrogen emission from the SN 1987A reverse shock," Astrophys. J. 743, 186 (2011).

Kevin France is professor of astrophysical and planetary sciences at the University of Colorado. $\mathrm{He}$ is the PI of the ESCAPE Small Explorer mission, the CUTE small satellite mission, and a NASA-supported sounding rocket program to develop critical path hardware for future astrophysics missions. He is a regular guest observer with Hubble and member of the LUVOIR STDT. He received his PhD from Johns Hopkins University and was awarded NASA's Nancy Grace Roman Fellowship.

Biographies of the other authors are not available. 